\title{
Künstliche Intelligenz muss dem Gemeinwohl dienen
}

\section{Eine verbraucherzentrierte KI verbindet Interessen der Zivilgesellschaft mit mehr Innovationskraft}

\author{
Die künstliche Intelligenz birgt große Potenziale für innovative Produkte, mehr \\ Verbraucherfreundlichkeit und einen höheren Lebensstandard. Gleichwohl müssen \\ Risiken, etwa die Diskriminierung und Irreführung von Verbraucher*innen sowie \\ der Missbrauch durch Algorithmen durch große Plattformen Einhalt geboten \\ werden. Politik, Verwaltung und bürgerschaftliches Engagement müssen sich \\ diesen Risiken ebenso annehmen wie Unternehmen. Es braucht dafür ein breites, \\ zivilgesellschaftliches Ökosystem für eine verbraucherzentrierte künstliche Intelligenz.
}

\section{Einführung}

Nicht erst die Covid-19-Pandemie hat die vielen Herausforderungen der digitalen Transformation weltweit offen gelegt . Trotz der Vorteile der Technologie erleben wir Auseinandersetzungen zwischen Staaten über Fragen wie etwa die angemessene Besteuerung großer Technologiekonzerne, die zunehmende Marktmacht oder auch die steigende Ungleichheit durch die zunehmende Automatisierung. Darüber hinaus geraten Verbraucher*innen und die $\mathrm{Zi}$ vilgesellschaft zunehmend unter Druck: Die Datensouveränität wird in der Welt der Apps zu einer immer größeren Herausforderung. Und ausgefeilte Technologien wie künstliche Intelligenz

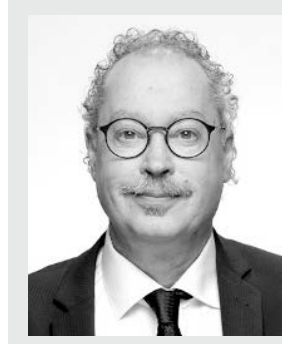

\section{Prof. Christian Kastrop}

ist Staatsekretär für Verbraucherschutz und Digitale Gesellschaft im Bundesministerium der Justiz und für Verbraucherschutz. Der studierte Ökonom lehrt an der FU Berlin. E-Mail: vorzimmer-st-kastrop@bmjv.bund.de

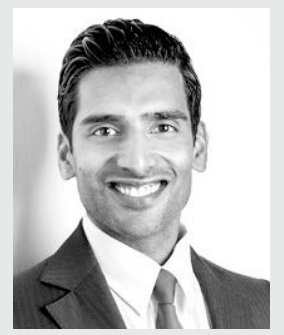

\section{Dr. Dominic Ponattu}

ist persönlicher Referent des Staatssekretärs im Bundesministerium der Justiz und für Verbraucherschutz. Er hat an der London School of Economics Volkswirtschaftslehre studiert und wurde in Mannheim promoviert. E-Mail: ponattu-do@bmjv.bund.de und Deep Learning haben dazu beigetragen, dass Desinformation und Manipulation nicht nur im Netz ein Thema sind, sondern auch eine erhebliche Bedrohung für Demokratien auf der ganzen Welt darstellen.

Zuletzt bemühten sich Regierungen und multilaterale Organisationen zu Recht um eine bessere Regulierung von digitalen Firmen und Diensten. Initiativen wie die der OECD zu „Base Erosion and Profit Shifting“ (BEPS) oder der Kampf gegen die globale Geldwäsche sind ein Beispiel dafür. Auch Verpflichtungen wie die Sustainable Development Goals (SDGs) tragen der Idee Rechnung, dass der Umgang mit (natürlichen) Ressourcen von größter Bedeutung ist, um Nachhaltigkeit und langfristigen Wohlstand in praktisch allen Ländern zu gewährleisten.

Neben der Regulierung von Unternehmen, Dienstleistungen und Ressourcen auf globaler Ebene müssen wir uns jedoch auch stärker um die Bewältigung eines anderen Wandels bemühen: den durch disruptive Technologien. Hier ist eine angemessene Steuerung mit vielen relevanten Beteiligten - neudeutsch Governance genannt - gefragt, damit diese Technologien Verbraucher ${ }^{\star}$ innen und der Zivilgesellschaft dienen - was letztlich der gesamten Wirtschaft zugutekommt. Die Governance für diese Technologien ist jedoch alles andere als trivial: Es gilt, die Risiken einer bestimmten Technologie wie KI zu beherrschen - und gleichzeitig die Vorteile dieser Technologie zu bewahren; idealerweise in einer Form, in der alle - und nicht nur einige wenige - in der Gesellschaft davon profitieren. In der Tat legt die ökonomische Forschung nahe, dass die Form und Qualität entscheidend für den Wohlstand sind - und dass dieser Weg dauerhaften Wohlstand bringen kann. 
Abbildung 1 | Geschätzte ökonomische Auswirkung der KI auf das BPI weltweit, nach Industriebranchen in 2030 (in \%), Quelle: Statista

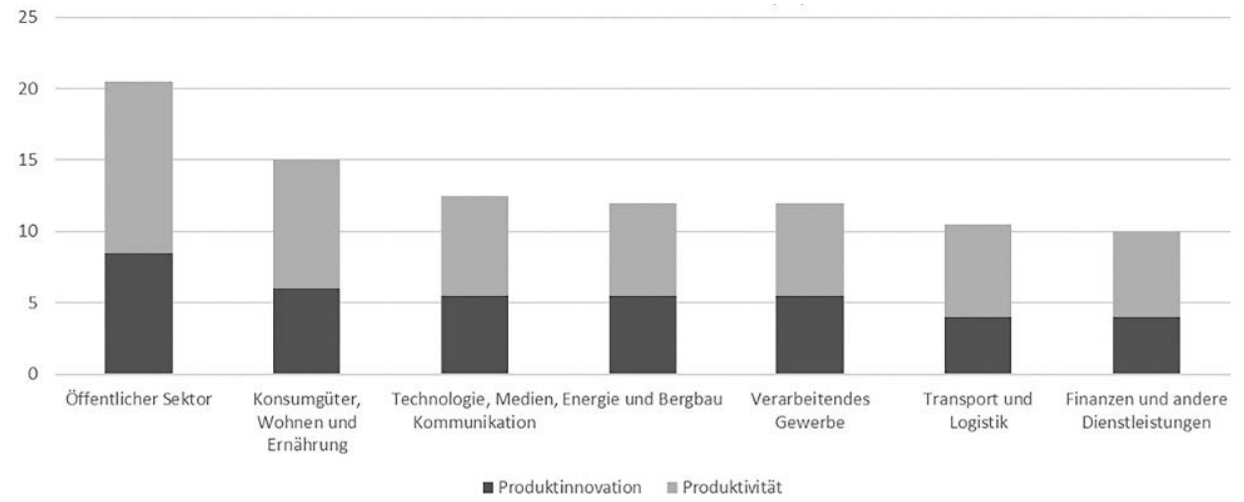

auch im Einzelhandel und sogar im Dienstleistungssektor (z.B. 10 Prozent bei Finanz- und Unternehmensdienstleistungen) sein werden. Zum einen werden diese Effekte durch neue, innovative Produkte erzielt - zum anderen durch Produktivitätswachstum, also ein effizienteres Zusammenspiel von Maschinen bzw. Equipment und der Arbeitskraft.

Zweifelsohne ist das erst einmal eine gute Nachricht - aber wir alle wissen: KI birgt auch ernsthafte Risiken, von denen einige bereits heute zutage getreten sind. Die Covid-19-Pandemie hat uns beide

\section{Künstliche Intelligenz als Lackmustest für gute digitale Governance}

Ein Beispiel dafür ist die künstliche Intelligenz (KI): Sie ist sicherlich eine der disruptivsten Technologien, die bereits jetzt einen weitreichenden Einfluss auf unser tägliches Leben und unsere Gesellschaft hat. Die Anwendungen reichen von der Sortierung von Nachrichten in unseren Suchmaschinen, über die Auswahl von Mitarbeiter*innen per Recruiting-Software, bis hin zur Diagnose und Behandlung von Krankheiten oder der Überwachung von Oppositionellen. Kein Bereich des privaten oder geschäftlichen Lebens bleibt unberührt. Kürzlich soll ein DeepMind-Algorithmus sogar das Problem der Proteinfaltung gelöst haben ein potenzieller Durchbruch für die Medikamentenentwicklung und eine echte Sensation.

Abbildung 1 deutet darauf hin, dass KI allein im Einzelhandel möglicherweise schon bald kaum wegzudenken ist: Die Durchdringung liegt bei etwa 1/3 im Einzelhandel in Branchen wie etwa der Bekleidung und Schuhe oder bei Lebensmitteln - was sich nicht nur auf die Beschäftigten in diesen Organisationen auswirken dürfte, sondern auch auf die Verbraucher*innen und somit natürlich auf den Verbraucherschutz.

Zudem wird in Abbildung 2 deutlich, dass KI einige bedeutende positive Wachstumseffekte für die Wirtschaft bewirken kann: KI wird sicherlich eine zusätzliche Quelle für Wachstum - und damit eine Erhöhung des Lebensstandards - sein. Die hier gezeigte Projektion legt nahe, dass die Wachstumseffekte durch KI besonders stark im öffentlichen Sektor (etwa 20 Prozent), aber

Abbildung 1 | Durchdringung von KI im Einzelhandel, weltweit in 2018, nach Einzelbereichen (in \%), Quelle: Statista

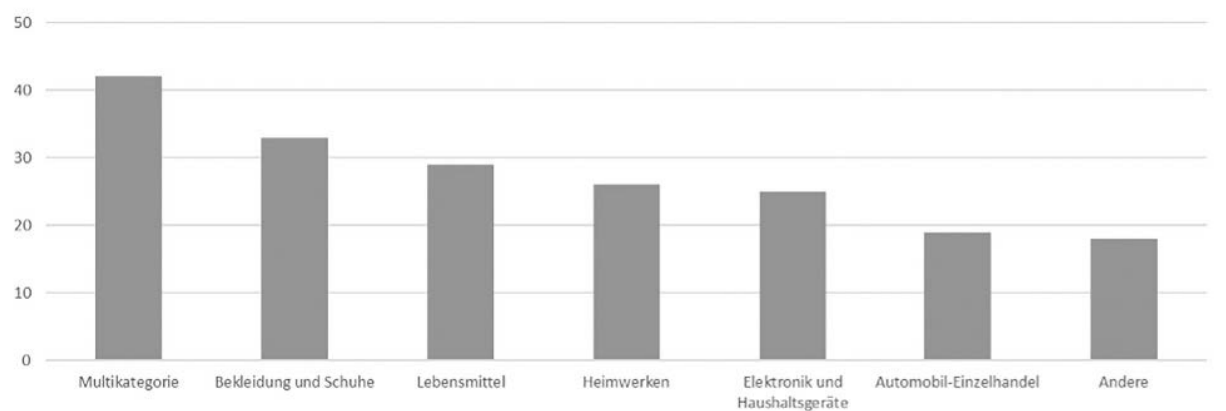

Gesichter gezeigt: Auf der einen Seite wird KI eingesetzt, um Risikogruppen zu identifizieren, Medikamente zu entwickeln und die Ausbreitung des Virus zu verhindern. Beispielsweise war es am 31. Dezember 2019 u.a. ein KI-System namens „Bluedot“, das vor einem Virusausbruch im chinesischen Wuhan warnte. Damit war der Algorithmus der WHO um ganze neun Tage voraus und hätte die globale Pandemie möglicherweise sogar schneller eindämmen oder gar verhindern können. Diese Beispiele verdeutlichen das beträchtliche Potenzial der KI-Technologie, das wir zum Wohle der Gesellschaft unbedingt fördern müssen.

Aus Sicht der Verbraucher ${ }^{*}$ innen kann aber dieselbe KI, die Menschenleben rettet, auch eine dunkle Seite zeigen. Nehmen wir zum Beispiel den sogenannten chinesischen „Health Code“, der von einer Telefon-App generiert wird. Dieser ist mittlerweile ein allgegenwärtiger Bestandteil des täglichen Lebens der chinesischen Bevölkerung. Dabei handelt es sich um einen Algorithmus, der nach der Analyse zahlreicher Standort- und Mobilfunkdaten das „Risiko einer Person“ errechnet. Ohne einen grünen Code vorweisen zu können, ist es in der Pandemie praktisch unmöglich, sich frei fortzubewegen Dies ermöglicht es den Behörden - mehr noch als früher - die Bewegungen einer Person zu verfolgen - das „Wo“, das „Wann“ und das „Mit wem“. Millionen von Kameras zur Gesichtserkennung, die die Bevölkerung auf Schritt und Tritt überwachen, tragen ebenfalls ihren Teil dazu bei.

Es sei angemerkt, dass diese Beispiele natürlich nicht auf Asien beschränkt sind. Auch in westlichen Demokratien beeinflussen KI-basierte Analyse- und Vorhersagetools schon seit einiger Zeit unseren Alltag. Es mag auf subtilere und unauffälligere Weise geschehen, aber es ist nicht weniger weitreichend. KI ist in der Lebenswirklichkeit der Menschen auch hierzulande angekommen. Man denke an „Pokémon Go“ al ein extremes Beispiel für eine scheinbar spielerische, aber potenziell manipulative Überwachungs-App. 


\section{Wege nach Vorn: Verbraucherzentrierte digitale Governance}

Die genannten Beispiele werfen Fragen auf, die sich neben Staat und Unternehmen vor allem die Zivilgesellschaft stellen muss: Möchte man hinnehmen, dass jede Bewegung, jede Reaktion, jede Äußerung und jeder Wunsch aufgezeichnet, katalogisiert und sogar manipuliert werden kann - oft ohne dass wir es bemerken? Wie wirkt sich das auf unser Recht auf Privatsphäre, auf die Ausübung unserer Menschenrechte und auf unsere demokratischen Freiheiten aus? Welche langfristigen Folgen hat es für unsere Demokratie, wenn die Algorithmen weniger Unternehmen darüber entscheiden, welche Informationen uns etwa vor einer Wahl angezeigt werden? Inwieweit ist unser Recht auf Informationsfreiheit eingeschränkt, wenn reale Medieninhalte auf der einen und synthetische, KI-manipulierte Inhalten auf der anderen Seite (z.B. Deepfakes) kaum unterscheidbar sind? Ganz zu schweigen von der viel diskutierten Gefahr, dass bestehende Ungleichheiten und Diskriminierungstendenzen durch KI-Anwendungen verstärkt werden könnten.

Richtig ist aber auch: Hinter KI und ihren Anwendungen stehen menschliche Entscheidungen - von Entwickler*innen, Programmierer*innen und den Unternehmen und Behörden, die KI einsetzen. Diese Entscheidungen brauchen einen (regulatorischen) Rahmen. Deshalb müssen wir uns - national und interna- tional - dafür einsetzen, angemessene und risikoadäquate Rahmenbedingungen für KI zu schaffen und einzuhalten. Diese müssen einerseits den Fortschritt durch KI nicht nur zulassen, sondern aktiv ermöglichen- allerdings nicht um jeden Preis. Innovation sollte nie Selbstzweck sein, sondern immer den Menschen und dem Gemeinwohl dienen - durch nachhaltiges Wirtschaftswachstum und damit höheren Lebensstandard. Kurzum: Wir brauchen eine besondere Form der digitalen Governance: diese muss verbraucherzentriert sein, um KI so zu regulieren, dass sie der Gesamtgesellschaft zugute kommen - und gleichzeitig Innovationen ermöglichen. Dies ist keineswegs ein Widerspruch ganz im Gegenteil: Wenn Verbraucherschutzstandards für KIAnwendungen hoch sind, vertrauen Bürger*innen entsprechenden Produkten, was wiederum die Akzeptanz der Technologien erhöht.

\section{Für eine verbraucherzentrierte KI zeigen wir zwei Beispiele auf, die einen Beitrag dazu leisten können.}

Zum einen ist die Schaffung eines rechtlichen Rahmens erforderlich. Um der Gefahr der Diskriminierung effektiv entgegenzuwirken, müssen wir konkrete Anforderungen an die von KI-Systemen verwendeten Daten - insbesondere Trainingsdaten - stellen. Die Autonomie des Menschen muss jederzeit gewährleistet sein - zum Beispiel durch menschliche Aufsicht, Eingriffsmöglich-

\section{Springer Vieweg}

\section{Testmanagement}

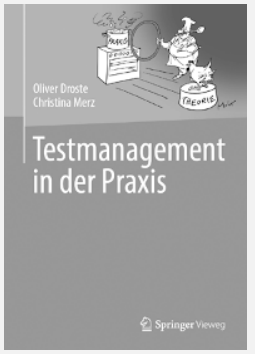

\author{
O. Droste, C. Merz \\ Testmanagement in der Praxis \\ 2019, XX, 230 S. 27 Abb., 17 Abb. in Farbe. Geb. \\ $€(D) 44,99|€(A) 46,25|{ }^{*}$ CHF 50.00 \\ ISBN 978-3-662-49652-7 \\ $€ 34,99 \mid{ }^{*} \mathrm{CHF} 40.00$ \\ ISBN 978-3-662-49653-4 (eBook)
}

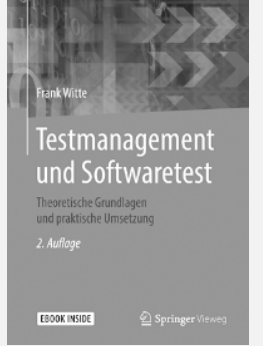

F. Witte

Testmanagement und Softwaretest Theoretische Grundlagen und praktische Umsetzung 2., erw. Aufl. 2019, XV, 300 S. 39 Abb. in Farbe. Book + eBook. Brosch. $€(D) 38,00|€(A) 39,77|{ }^{*} \mathrm{CHF} 42.00$ ISBN 978-3-658-25086-7 $€ 29,99 \mid{ }^{*} \mathrm{CHF} 33.50$ ISBN 978-3-658-25087-4 (eBook)

\section{Ihre Vorteile in unserem Online Shop: \\ Über 280.000 Titel aus allen Fachgebieten | eBooks sind auf allen Endgeräten nutzbar Kostenloser Versand für Printbücher weltweit}

$€(D)$ : gebundener Ladenpreis in Deutschland, $€(A)$ : in Österreich. ${ }^{*}$ : unverbindliche Preisempfehlung. Alle Preise inkl. MwSt. 
keiten und abschließende Entscheidungen. Bei KI-beeinflussten Entscheidungen muss der Betroffene die gleichen Widerspruchsund Rechtsschutzmöglichkeiten haben wie bei rein menschlichen Entscheidungen.

Die Bundesregierung - und hier das Bundesministerium der Justiz und für Verbraucherschutz - hat sich gemeinsam mit anderen Ländern im Europarat im Ad-hoc-Ausschuss für künstliche Intelligenz (CAHAI) zusammengeschlossen. Das Komitee hat auf Basis breiter Multi-Stakeholder-Konsultationen die Machbarkeit und die möglichen Elemente eines rechtlichen Rahmens für die Entwicklung, Gestaltung und Anwendung von künstlicher Intelligenz untersucht, der auf den Standards des Europarats zu Menschenrechten, Demokratie und Rechtsstaatlichkeit basiert. Daraus ist eine Machbarkeitsstudie entstanden, die mögliche Elemente eines spezifischen Rechtsrahmens für KI skizziert. ${ }^{1}$ Die Studie zeigt: Die Europäische Union hat einen starken Hebel, um diese Debatte zu gestalten: Das Legislativpaket über digitale Dienste („Digital Services/Markets Act“), das im Dezember 2020 vorgelegt wurde, sowie der Rechtsakt über künstliche Intelligenz, der im April dieses Jahres vorgestellt wird, bieten die Möglichkeit, den rechtlichen und somit den Governance-Rahmen für KI zu gestalten.

Ein zweites Beispiel für eine menschenzentrierte digitale Governance ist ein sogenanntes „Ökosystems“ für KI-Akteure. Schließlich geht es beim Umgang mit den Risiken und Vorteilen von KI auch darum, Dialog und Zusammenarbeit von mindestens drei Gruppen zu vereinen: diejenigen, die den Code entwickeln, diejenigen, die ihn in Produkten implementieren - und die Betroffenen in der Zivilgesellschaft, die Anwendungen als Verbraucher ${ }^{*}$ innen nutzen bzw. diesen ausgesetzt sind. Wie viele andere Länder versucht auch Deutschland, diese Art von Ökosystemen zu schaffen. So plant das Bundesministerium der Justiz und für Verbraucherschutz den Aufbau eines Netzwerks für verbraucherzentrierte KI, in dem die oben genannten Gruppen zusammenkommen, um über den fairen und angemessenen Einsatz von KI sowie den erforderlichen Rechtsrahmen beraten. Entwickler und Umsetzer sollten mit den Interessen der heterogenen Zivilgesellschaft konfrontiert werden - und umgekehrt -, so dass ein gegenseitiger Austausch der Belange aller Beteiligten stattfindet. Denn eine so tiefgreifende Technologie wie KI geht alle etwas an.

\section{Fazit: Zivilgesellschaft als Schlüssel}

Die Regulierung von KI ist ein gutes Beispiel dafür, warum eine auf Verbraucher ${ }^{\star}$ innen - und somit auf den Menschen - aus-

1 Machbarkeitsstudie Entwicklung gemäß den Standardsdes Europarats, die vom CAHAl-Ausschuss angenommen wurden. Abrufbar hier: https://rm.coe.int/ cahai-2020-23-final-eng-feasibility-study-/1680a0c6da gerichtete digitale Governance von zentraler Bedeutung ist. Sie kann dabei helfen, den technologischen Umbruch so zu gestalten, dass die Vorteile jedem Mitglied der Gesellschaft zugänglich sind - während gleichzeitig Risiken wie Diskriminierung und Desinformation effektiv entgegengesteuert werden können. Zwei Säulen sind dabei zentral für eine verbraucherzentrierte digitale Governance: Erstens brauchen wir einen rechtlichen Rahmen, der einer Technologie wie der KI angemessene Grenzen und Regeln setzt. Zweitens brauchen wir eine breit angelegte Diskussion zwischen allen Beteiligten in der Gesellschaft, um die Vorzüge und Risiken einer bestimmten Technologie zu erörtern - dieser Prozess kann der Schlüssel zur Schaffung von Legitimität für den Einsatz einer technologischen Disruption wie KI sein.

Es ist klar, dass diese Form der Governance auch in anderen Bereichen der Digitalpolitik zum Tragen kommen kann. Zum Beispiel ist die Frage der Datenzugänge und des Datenschutzes eine, die ebenfalls eine sorgfältige Abwägung der Risiken und Vorteile einer kohärenten Datenstrategie erfordert. Auch hier hat die Covid-19-Pandemie gezeigt, dass diese Abwägungen alles andere als einfach sind. Gibt es einen Kompromiss zwischen Datenschutz und intelligenter Nutzung von Daten zur Pandemiebekämpfung? Welche Art von Daten sollten verwendet werden, um Entscheidungen über Lockdowns zu treffen? Welche Art von Datenintermediären sind die richtigen, um Verbraucher- und Firmendaten zu schützen? Fragen dieser Art erfordern auch klare Rahmenbedingungen für die Datennutzung und eine gesellschaftliche Abwägung, wie weit wir die Nutzung von Daten bei großen gesellschaftlichen Entscheidungen gehen lassen wollen.

Klar sollte aus unserer Sicht jedenfalls sein: Die Zivilgesellschaft ist der Grundstein für diese Überlegungen. Wir schlagen daher vor, nicht nur die digitalen Bürgerrechte gegenüber Plattformen zu fördern, sondern auch der Zivilgesellschaft eine starke, institutionelle Stimme bei der Gestaltung der digitalen Rechte in der Gesellschaft zu geben. Für eine verbraucherzentrierte digitale Governance braucht es daher aus unserer Sicht starke NGOs, Ethikräte und andere zivilgesellschaftliche Organisationen, die ebenso in die Digitalpolitik eingebunden sind wie Unternehmen und der Staat. So kann pluralistische Digitalpolitik gelingen.

\section{Literatur}

[1] Acemoglu, D., Johnson, S. and Robinson, J.A., 2001. The colonial origins of compara-tive develop-ment: An empirical investigation. American Economic Review, 91(5), pp.1369-1401.

[2] AD HOC COMMITTEE ON ARTIFICIAL INTELLIGENCE (CAHAI), Feasibility study on a legal framework on AI design, development and application based on Council of Europe's standards adopted by the CAHAI on $17 \mathrm{De}-$ cember 2020. Abrufbar hier: https://rm.coe.int/cahai-2020-23-final-engfeasibility-study-/1680a0c6da 